\title{
GASTO DE BOLSILLO EN SALUD EN ADULTOS MAYORES PERUANOS: ANÁLISIS DE LA ENCUESTA NACIONAL DE HOGARES SOBRE CONDICIONES DE VIDA Y POBREZA 2017
}

\author{
Akram Hernández-Vásquez ${ }^{1, a}$, Carlos Rojas-Roque ${ }^{2, b}$, Marilina Santero ${ }^{3,4, c}$, \\ Francisco Javier Prado-Galbarro ${ }^{5, \mathrm{~d}}$, Diego Rosselli 6,e
}

\author{
RESUMEN
}

Objetivos. Estimar el gasto de bolsillo en salud (GBS) e identificar sus factores asociados en adultos mayores peruanos. Materiales y métodos. Estudio transversal analítico de la Encuesta Nacional de Hogares sobre Condiciones de Vida y Pobreza (ENAHO) 2017. Se consideró como adulto mayor a todo individuo de 60 y más años y el GBS como variable principal de estudio. Se estimaron razones de prevalencia (RP) y razones de prevalencia ajustada ( $R P a)$ para cada uno de los factores asociados a GBS. Las medias del GBS fueron estimadas mediante un modelo lineal generalizado con distribución gamma y función de enlace log. Todos los intervalos de confianza (95\%) de los estimadores fueron calculados mediante bootstrapping con el método basado en la normal. Resultados. Se incluyeron 18386 adultos mayores, de los cuales en el 56,5\% se reportó GBS. La media y mediana del GBS es de 140,8 (USD 43,2) y 34,5 (USD 10,6) soles, respectivamente. Factores como procedencia urbana, mayor nivel de educación, padecer enfermedades crónicas y mayores gastos per cápita aumentan hasta 1,6 veces la probabilidad de GBS. En los afiliados al Seguro Integral de Salud (SIS) se reduce el GBS en 63,0 soles (USD 19,3) comparado con aquellos sin ningún seguro de salud. Conclusiones. Seis de cada diez adultos mayores peruanos reportó GBS para atender su salud. Esto genera inequidad en el acceso a los servicios de salud, principalmente para los grupos socialmente vulnerables. Se sugiere investigar el impacto económico de los seguros sanitarios y el abordaje preventivo-promocional de las enfermedades crónicas, en aras de reducir el GBS y mejorar la eficiencia del sistema de salud peruano.

Palabras clave: Economía médica; Encuesta de salud; Servicios de salud; Adulto mayor; Perú (fuente: DeCS BIREME).

\section{HEALTH-RELATED OUT-OF-POCKET EXPENSES IN OLDER PERUVIAN ADULTS: ANALYSIS OF THE NATIONAL HOUSEHOLD SURVEY ON LIVING CONDITIONS AND POVERTY 2017}

\begin{abstract}
Objectives. To estimate out-of-pocket spending on health (GBS) and identify its associated factors in Peruvian older adults. Materials and Methods. Analytical cross-sectional study of the National Household Survey on Living Conditions and Poverty (ENAHO) 2017. Older adults were considered to be all individuals aged 60 and over and the GBS was considered the main study variable. Prevalence ratios (PR) and adjusted prevalence ratios (PRa) were estimated for each of the factors associated with GBS. GBS means were estimated using a generalized linear model with gamma distribution and log binding function. All confidence intervals (95\%) of the estimators were calculated by bootstrapping with the normal-based method. Results. Eighteen 386 older adults were included, of which 56.5\% reported GBS. The mean and median GBS is S/. 140.8 (USD 43.2 ) and S/. 34.5 (USD 10.6), respectively. Factors such as urban origin, a higher level of education, chronic diseases and higher per capita expenses increase the probability of GBS by up to 1.6 times. In those affiliated to the Integral Health Insurance (SIS), the GBS is reduced by 63.0 soles (USD 19.3) compared to those without any health insurance. Conclusions. Six out of ten older Peruvian adults reported GBS to attend to their health needs. This generates an access inequity in terms of health services, mainly for socially-vulnerable groups. We suggest researching into the economic impact of health insurance and the preventive-promotional approach to chronic diseases, in order to reduce GBS and improve the efficiency of the Peruvian health system.
\end{abstract}

Keywords: Medical economics; Health survey; Health Services; Older adult; Perú (source: MeSH NLM).

\footnotetext{
Centro de Excelencia en Estudios Económicos y Sociales, Universidad San Ignacio de Loyola. Lima, Perú.

CIDS, Centro de Investigación en Demografía y Salud. León, Nicaragua.

Universidad de Buenos Aires. Buenos Aires, Argentina.

Instituto de Efectividad Clínica y Sanitaria (IECS). Buenos Aires, Argentina.

Centro de Salud Poblacional, Instituto Nacional de Salud Pública. Cuernavaca, México.

Departamento de Epidemiología Clínica y Bioestadística, Facultad de Medicina, Pontificia Universidad Javeriana. Bogotá, Colombia.

Médico cirujano, magíster en Gestión y Políticas Públicas; ${ }^{b}$ licenciado en Economía; ${ }^{\mathrm{c}}$ médica, especialista en Epidemiología, magíster en Efectividad

Clínica; ${ }^{\mathrm{d}}$ licenciado en Estadística, doctor en Ciencias Médicas; ${ }^{\mathrm{e}}$ médico, especialista en Neurología, magíster en Educación

Recibido: 18/07/2018 Aprobado: 12/09/2018 En línea: 25/09/2018
}

Citar como: Hernández-Vásquez A, Rojas-Roque C, Santero M, Prado-Galbarro FJ, Rosselli D. Gasto de bolsillo en salud en adultos mayores peruanos: análisis de la Encuesta Nacional de Hogares sobre Condiciones de Vida y Pobreza 2017. Rev Peru Med Exp Salud Publica. 2018;35(3):390-9. doi: 10.17843/ rpmesp.2018.353.3815. 


\section{INTRODUCCIÓN}

El gasto de bolsillo en salud (GBS) se define como el pago directo no reembolsable realizado por las personas que hacen uso de servicios de salud y que no son financiados por el Estado o los seguros de salud (1). El GBS puede ser parte de tarifas y copagos para generar ingresos, racionalizar el uso de los servicios de salud, contener los costos, reflejar vacíos en la cobertura del sistema sanitario, mejorar la eficiencia del sistema sanitario y la calidad del servicio ${ }^{(1)}$. El GBS puede desalentar la utilización y convertirse en una barrera de acceso a los servicios de salud, así como exponer al riesgo de empobrecimiento por gastos de atención de problemas de salud, especialmente en países en vías de desarrollo.

Según la Organización Mundial de la Salud (OMS), el GBS del hogar se ha duplicado en América Latina, pasando de USD 55,2 millones en el 2000 a 122,5 millones en $2015^{(2)}$. Ante el crecimiento acelerado de la población adulta mayor, se viene prestando atención al análisis del GBS por el riesgo financiero de esta población dado el uso incrementado de servicios de salud y alta prevalencia de enfermedades crónicas no transmisibles (ECNT) ${ }^{(3,4)}$. Así, los sistemas sanitarios se enfrentan a grandes desafíos para lograr la equidad en el financiamiento y la protección frente al riesgo financiero de los adultos mayores más vulnerables.

Se han encontrado diversos factores que se asocian al GBS, como por ejemplo, sexo, edad, área de residencia, nivel de educación, ingresos, presencia de enfermedades crónicas y disponer de un seguro de salud ${ }^{(5-12)}$. Así, los adultos mayores con bajos ingresos tienen una mayor proporción de GBS en relación con sus ingresos, un menor nivel educativo se asocia con un mayor GBS, la falta de cobertura de un seguro de salud y el sexo femenino presentan GBS más alto debido a sus menores ingresos y menor tasa de participación laboral ${ }^{(13)}$. Del mismo modo, la presencia de tres o más enfermedades crónicas puede incrementar diez veces el GBS anual por la compra de medicamentos ${ }^{(3)}$. A diferencia de las personas más jóvenes, los adultos mayores se enfrentan a una reducción de su potencial productivo, multicomorbilidades y polimedicación, lo cual podría incurrir en un mayor GBS y posterior empobrecimiento por gastos en salud.

En Perú, los dos últimos censos indican que la población de 60 y más años se incrementó, pasando de 9,1 \% en 2007 a $11,9 \%$ en 2017. Asimismo, el número de adultos mayores dependientes pasó de 15,1 a 19,3 por cada 100 personas en edad de trabajar ${ }^{(14)}$. Dada la poca información existente sobre el GBS en adultos mayores peruanos y considerando el contexto de envejecimiento demográfico, la transición epidemiológica, y las posibles dificultades en el acceso a los servicios de salud, es relevante estudiar la magnitud de GBS debido al impacto negativo en la economía de los adultos mayores peruanos que puede conllevar.

\section{MENSAJES CLAVE}

Motivación para realizar el estudio. Ante el crecimiento acelerado de la población adulta mayor, los sistemas de salud se enfrentan a grandes desafíos para lograr la equidad en la protección frente al riesgo financiero de los más vulnerables.

Principales hallazgos. Los adultos mayores en Perú pagan de su bolsillo para atender su salud, principalmente aquellos con menor nivel educativo, menor gasto per cápita, o que padecen enfermedades crónicas.

Implicancias. Resta investigar el impacto económico de los seguros sanitarios y el abordaje preventivo-promocional de las enfermedades crónicas en aras de reducir el gasto de bolsillo en salud y mejorar la eficiencia del sistema de salud peruano.

En tal sentido, el presente estudio tiene como objetivo estimar el GBS e identificar sus factores asociados en adultos mayores peruanos.

\section{MATERIALES Y MÉTODOS}

\section{DISEÑO Y POBLACIÓN DE ESTUDIO}

Se realizó un estudio transversal analítico de una submuestra de la Encuesta Nacional de Hogares sobre Condiciones de Vida y Pobreza (ENAHO) 2017, realizada por el Instituto Nacional de Estadística e Informática del Perú (INEI). La submuestra estuvo conformada por personas de 60 y más años según la Ley 30490, Ley de la Persona Adulta Mayor ${ }^{(15)}$. La ENAHO es una encuesta anual de carácter transversal que emplea un muestreo probabilístico, de áreas, estratificado, multietápico e independiente con representatividad a nivel nacional, departamental, región natural y urbano/rural. La encuesta recaba información acerca de las condiciones de vida de la población peruana. La población de estudio de la ENAHO está definida como el conjunto de todas las viviendas particulares y sus ocupantes residentes en el área urbana y rural del país. Las bases de datos de la ENAHO son de libre disposición y pueden ser obtenidas del portal web del INEI (http://iinei.inei.gob.pe/ microdatos).

\section{VARIABLES Y MEDICIONES}

Se consideró el GBS durante el 2017 como variable principal de estudio. La variable fue construida sumando los pagos desembolsados (en soles corrientes) por el adulto mayor o algún miembro de su hogar, para los siguientes servicios de salud, identificados por sus códigos en la encuesta: consulta médica (p41601), medicamentos (p41602), análisis (p41603), rayos X (p41604), exámenes (p41605), servicio dental y conexos (p41606), servicio oftalmológico 
(p41607), compra de lentes (p41608), vacunas ( $p 41609)$ otros gastos (p41612), y hospitalización (p41613). El periodo de referencia para los pagos desembolsados fue de cuatro semanas para consulta médica, medicamentos, análisis, rayos $\mathrm{X}$ y exámenes; de tres meses para servicio oftalmológico, compra de lentes, servicio dental y conexos, vacunas y otros gastos; y de un año para hospitalización.

Las siguientes variables junto con sus códigos en la encuesta fueron incluidas como factores que predisponen el uso del sistema de salud y por tanto, mayores probabilidades de efectuar un GBS, según el modelo teórico de Andersen y Newman (16): sexo (p207), edad (p208a), estado civil (p209), área de residencia, educación (p301a), tipo de seguro médico (p4191, p4192, p4194, p4195), dominio, padecimiento de alguna enfermedad crónica (p401), sufrimiento de alguna limitación física o psicológica (p401h1 a p401h6) y quintiles de gasto del hogar per cápita mensual.

Las edades fueron agrupadas en decenios y se consideró área de residencia rural si el estrato muestral tuvo menos de 401 viviendas o la casa pertenece al empadronamiento rural; en caso contrario, se categorizó área de residencia urbana. La variable afiliación a un tipo de seguro de salud incluyó las categorías "Sin seguro», «Sanidad de las Fuerzas Armadas y Policía Nacional (FF.AA.)», «Seguro Integral de Salud (SIS)», "Seguro Social (EsSalud)» y «Privado o Entidades Prestadoras de Salud (EPS)». En el Perú, los servicios de salud de la FF.AA. brinda cobertura de salud a los militares, policías y sus familiares; el SIS pertenece al sector público y subsidia los servicios de salud a la población no asegurada que vive en condiciones de pobreza y pobreza extrema; por su parte, EsSalud corresponde a la seguridad social y brinda servicios de salud a los trabajadores afiliados y sus derechohabientes; $\mathrm{y}$, finalmente, los privados o EPS conforman la provisión privada de servicios de salud ${ }^{(17)}$.

El padecimiento de una enfermedad crónica y de alguna limitación física o psicológica fue obtenido mediante autorreporte (Sí/No). Para evaluar el padecimiento de alguna limitación física o psicológica, se creó una variable dicotómica que fue sí cuando el adulto mayor reportaba al menos una limitación, y no en caso de que no reportaba ninguna. Los quintiles de gasto del hogar per cápita mensual se obtuvieron dividiendo el gasto total bruto anual del hogar entre 12 y luego dividiéndolo entre el número de miembros del hogar encuestado. Los quintiles de gasto del hogar per cápita mensual es una variable ordinal que se incluyó como una aproximación al nivel de riqueza de los adultos mayores y de las familias.

\section{ANÁLISIS ESTADÍSTICO}

La integración de bases de datos, procesamiento y análisis estadístico se realizó mediante el software estadístico Stata® v14.2 (Stata Corporation, College Station, Texas, USA), especificándose las características del diseño muestral de la encuesta que incluyó los factores de expansión según grupos etarios (factor07a) de la ENAHO, para lo cual se utilizó el comando svy. Para todas las pruebas estadísticas se consideró un valor de $p<0,05$ como estadísticamente significativo.

Las características sociodemográficas fueron descritas mediante frecuencias absolutas y proporciones ponderadas. Los niveles de GBS se reportaron en medianas y rango intercuartílico, dada la no distribución normal de la variable, y se estimaron y compararon las medias ponderadas de GBS para aquellos adultos mayores que tuvieron un GBS mayor que cero. Las diferencias de medianas ponderadas se obtuvieron mediante regresión de quintiles y las diferencias en las medias ponderadas se evaluaron mediante la prueba t de Student o ANOVA para el caso de variables con más de dos categorías, tomando en consideración los factores de expansión de la ENAHO.

Para evaluar los factores asociados al GBS se recodificó el GBS en una variable dicotómica que toma valores de uno cuando el adulto mayor reportó GBS y cero en caso contrario. Seguidamente, se estimaron razones de prevalencia (RP) mediante un análisis bivariado para cada una de las variables incluidas en el estudio. Asimismo, se estimaron razones de prevalencia ajustadas ( $R P a)$ en aquellos factores que arrojaron un valor $p<0,20$ en el análisis bivariado. Todas las estimaciones se realizaron usando un modelo lineal generalizado con distribución Poisson y función de enlace log para muestras complejas ${ }^{(18)}$.

Para describir los niveles de gasto de GBS, se estimó un modelo lineal generalizado con distribución gamma y función de enlace log. Se eligió esta distribución debido a que los valores de gasto están positivamente sesgados por la presencia de datos cercanos a cero ${ }^{(19)}$. La variable dependiente GBS es numérica, medida en soles corrientes. Las estimaciones se realizaron de forma bivariada con todas aquellas variables que resultaron asociadas al GBS. Los coeficientes $\beta$ resultantes fueron posteriormente analizados mediante predictores marginales, con el objetivo de obtener resultados interpretables en soles corrientes. Para hacer comparaciones internacionales se convirtieron los soles corrientes en dólares estadounidenses. El tipo de cambio promedio (S/ por USD) empleado para el periodo 2017 fue $3,26^{(20)}$.

Todos los intervalos de confianza (95\%) de los estimadores fueron calculados mediante bootstrapping con el método basado en la normal, incluyendo las ponderaciones y el efecto de diseño propio del muestreo complejo de la encuesta, para corregir residuos potencialmente distribuidos de forma diferente de lo que supone el modelo. El bootstrapping se efectuó con 1000 replicaciones, tal como se recomienda en economía de la salud ${ }^{(21)}$. 


\section{CONSIDERACIONES ÉTICAS}

La realización del estudio no requirió la aprobación de un comité de ética por tratarse de un análisis de datos secundarios que son de dominio público y que no permite identificar a los individuos encuestados.

\section{RESULTADOS}

Se incluyeron un total de 18386 adultos mayores (población expandida 3355 037), luego de excluir observaciones por no cumplir criterios de selección o tener datos faltantes (Figura 1). La mayoría de los encuestados son del sexo femenino $(52,5 \%)$, con una edad entre 60 y 69 años $(54,8 \%)$, casados o convivientes $(60,0 \%)$, cuatro de cada cinco residen en el área urbana $(79,4 \%)$ y cerca de la mitad tiene educación primaria (42,7\%). Aproximadamente una quinta parte carece de seguro de salud $(18,3 \%)$, mientras que $41,6 \%$ indica estar afiliado al SIS y $36,8 \%$ al seguro de EsSalud. La mayoría de los adultos mayores reportaron padecer una enfermedad crónica $(75,8 \%)$ y $14,5 \%$ de alguna limitación física o psicológica (Tabla 1).

Del total de adultos mayores incluidos en el análisis, 9842 (56,5 \%) reportaron GBS. La mayor proporción de adultos mayores que reportaron GBS se presenta en los que están afiliados al seguro privado o de entidades prestadoras de salud (EPS) $(71,0 \%)$ y los que se ubican en el quintil más rico de gasto per cápita $(68,0 \%$ ) (Tabla 1$)$.

La media y la mediana de GBS de los adultos mayores es de 140,8 (USD 43,2) y 34,5 soles (USD 10,6) respectivamente. Se encontraron diferencias en el nivel de GBS según los factores que predisponen al GBS, a excepción del sexo y el padecimiento de alguna limitación física o psicológica. El mayor nivel de GBS lo reportan los adultos mayores del quintil más rico de gasto per cápita (293,3 soles equivalentes a USD 90,0), quienes están afiliados al seguro privado o EPS $(249,9$ soles equivalentes a USD 76,6$)$ y quienes tienen educación superior ( 247,9 soles equivalentes a USD $76,0)$. Se reportó también que los mayores niveles de GBS ocurren en Lima Metropolitana (183,5 soles equivalentes a USD 56,3$)$ seguido del resto de costa $(131,5$ soles equivalentes a USD 40,3). En cambio, quienes reportan menor nivel de GBS son los adultos mayores del primer y segundo quintil de gasto per cápita ( 35,7 y 59,0 soles respectivamente o USD 11,0 y USD 18,1, respectivamente), los que tienen nivel inicial o ningún nivel de educación ( 68,0 soles equivalentes a USD 20,9) y los que habitan en zonas rurales $(70,5$ soles equivalentes a USD 21,6$)$ (Tabla 2$)$.

La Tabla 3 reporta los factores asociados al GBS y las estimaciones de los niveles medios de GBS. En el modelo

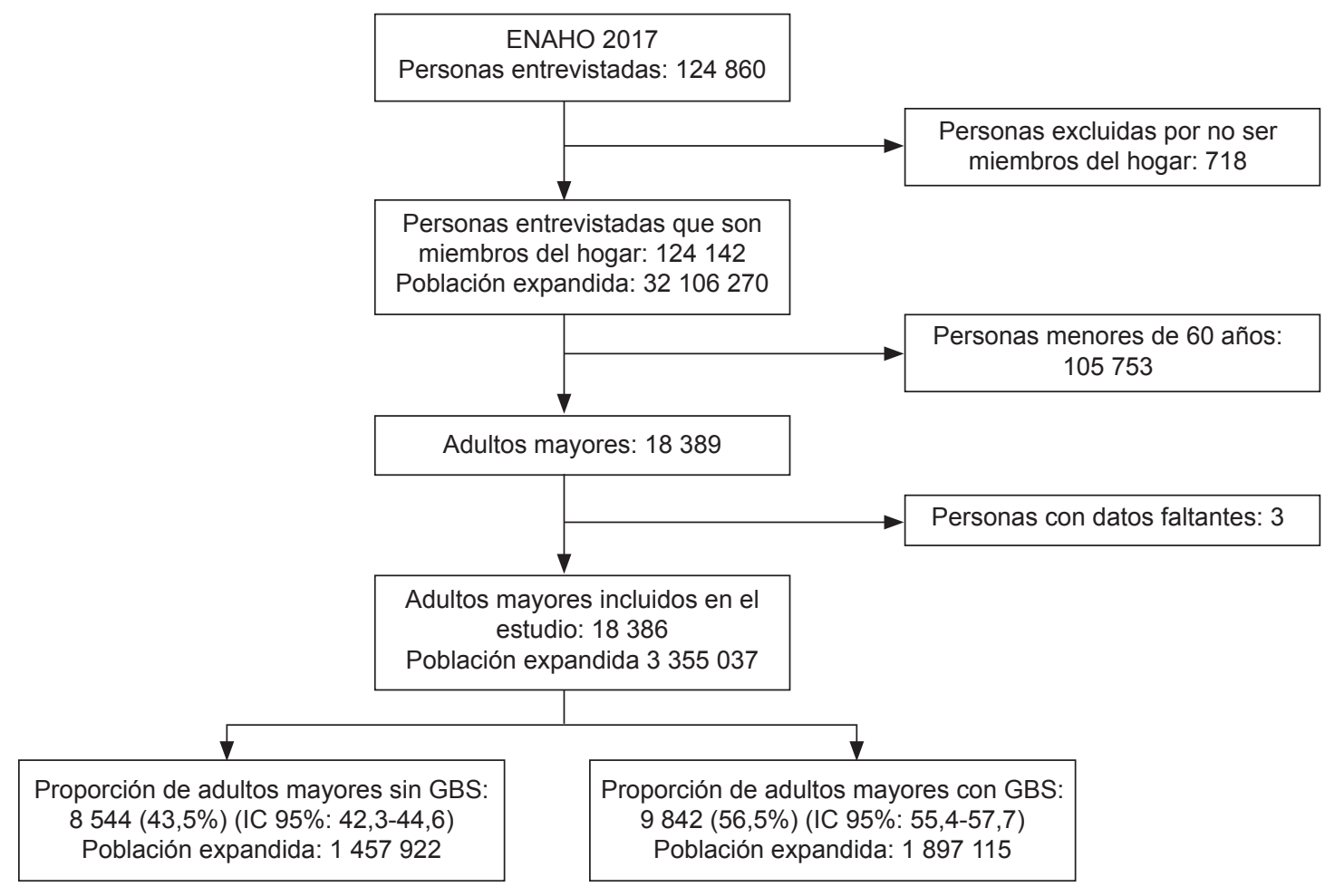

Figura 1. Flujograma de la selección de adultos mayores incluidos en el estudio

ENAHO: Encuesta Nacional de Hogares sobre Condiciones de Vida y Pobreza GBS: gasto de bolsillo en salud 
Tabla 1. Características de los adultos mayores peruanos y reporte de gasto de bolsillo en salud, Encuesta Nacional de Hogares sobre Condiciones de Vida y Pobreza, 2017

\begin{tabular}{|c|c|c|c|c|}
\hline \multirow{2}{*}{ Características } & \multicolumn{2}{|c|}{$\begin{array}{l}\text { Adultos mayores encuestados } \\
(\mathrm{N}=18 \mathrm{386})\end{array}$} & \multicolumn{2}{|c|}{ Gasto de bolsillo en salud ${ }^{\dagger}$} \\
\hline & $\begin{array}{c}\text { Frecuencia } \\
\text { absoluta }\end{array}$ & $\begin{array}{l}\text { Proporción } \\
\text { ponderada* }\end{array}$ & $\mathbf{n}$ & $\begin{array}{l}\text { Proporción } \\
\text { ponderada* }\end{array}$ \\
\hline \multicolumn{5}{|l|}{ Sexo } \\
\hline Hombre & 8808 & 47,5 & 4480 & 53,4 \\
\hline Mujer & 9578 & 52,5 & 5362 & 59,4 \\
\hline \multicolumn{5}{|l|}{ Grupo etario (años) } \\
\hline $60-69$ & 9371 & 54,8 & 5022 & 56,8 \\
\hline $70-79$ & 5771 & 28,5 & 3142 & 57,9 \\
\hline $80-89$ & 2720 & 14,0 & 1394 & 52,9 \\
\hline 90 o más & 524 & 2,7 & 284 & 55,8 \\
\hline \multicolumn{5}{|l|}{ Estado civil } \\
\hline Casado(a) o conviviente & 11313 & 60,0 & 5860 & 54,3 \\
\hline Soltero(a) & 887 & 5,9 & 448 & 55,6 \\
\hline Viudo(a), divorciado(a) o separado(a) & 6186 & 34,1 & 3534 & 60,7 \\
\hline \multicolumn{5}{|l|}{ Área de residencia } \\
\hline Urbano & 11445 & 79,4 & 6741 & 59,4 \\
\hline Rural & 6941 & 20,6 & 3101 & 45,6 \\
\hline \multicolumn{5}{|l|}{ Nivel educativo } \\
\hline Sin nivel o inicial & 3698 & 15,8 & 1668 & 48,4 \\
\hline Primaria & 8449 & 42,7 & 4446 & 56,0 \\
\hline Secundaria & 3683 & 24,3 & 2178 & 59,8 \\
\hline Superior & 2556 & 17,2 & 1550 & 60,9 \\
\hline \multicolumn{5}{|l|}{ Tipo de seguro de salud } \\
\hline Sin seguro & 3098 & 18,3 & 1745 & 58,8 \\
\hline FF.AA. & 331 & 2,2 & 222 & 67,5 \\
\hline SIS & 8972 & 41,6 & 4435 & 53,7 \\
\hline EsSalud & 5857 & 36,8 & 3369 & 57,6 \\
\hline Privado o EPS & 128 & 1,1 & 71 & 71,0 \\
\hline \multicolumn{5}{|l|}{ Dominio geográfico } \\
\hline Lima Metropolitana & 2456 & 36,0 & 1493 & 60,8 \\
\hline Resto de costa & 5740 & 24,2 & 3314 & 58,4 \\
\hline Sierra & 7354 & 30,5 & 3574 & 51,0 \\
\hline Selva & 2836 & 9,3 & 1461 & 53,2 \\
\hline \multicolumn{5}{|l|}{ Enfermedades crónicas } \\
\hline Sí & 14021 & 75,8 & 8127 & 61,3 \\
\hline No & 4365 & 24,2 & 1715 & 41,5 \\
\hline \multicolumn{5}{|l|}{ Limitaciones físicas o psicológicas } \\
\hline Sí & 2997 & 14,5 & 8255 & 56,6 \\
\hline No & 15389 & 85,5 & 1587 & 56,0 \\
\hline \multicolumn{5}{|l|}{ Quintiles de gasto per cápita } \\
\hline Quintil 1 (menor) & 5069 & 20,0 & 1953 & 41,0 \\
\hline Quintil 2 & 4023 & 20,0 & 2126 & 53,5 \\
\hline Quintil 3 & 3317 & 20,0 & 1929 & 59,6 \\
\hline Quintil 4 & 3109 & 20,0 & 1886 & 60,7 \\
\hline Quintil 5 (mayor) & 2868 & 20,0 & 1948 & 68,0 \\
\hline
\end{tabular}

*Se incluyó el factor de expansión y especificaciones muestrales de la ENAHO 2017

† Número y proporción ponderada por cada categoría de las características incluidas

FF.AA.: Sanidad de las Fuerzas Armadas y Policía Nacional

SIS: Seguro Integral de Salud

EsSalud: Seguro Social

EPS: Entidades Prestadoras de Salud 
Tabla 2. Gasto de bolsillo en salud en adultos mayores peruanos (soles corrientes), Encuesta Nacional de Hogares sobre Condiciones de Vida y Pobreza, 2017

\begin{tabular}{|c|c|c|c|c|}
\hline Variable & Mediana (Q1-Q3) & Valor de $p$ & Media (IC 95\%) ${ }^{\ddagger}$ & Valor de $p$ \\
\hline Población total & $34,5(9,5-128,0)$ & & $140,8(130,4-151,2)$ & \\
\hline Sexo & & 0,212 & & 0,108 * \\
\hline Hombre & $31,0(9,0-120,0)$ & & $132,1(118,6-145,5)$ & \\
\hline Mujer & $35,0(10,0-145,0)$ & & $147,9(133,2-162,5)$ & \\
\hline Grupo etario (años) & & $<0,001$ & & $<0,001^{\dagger}$ \\
\hline $60-69$ & $30,0(8,0-120,0)$ & & $129,0(116,9-141,0)$ & \\
\hline $70-79$ & $35,5(10,0-132,0)$ & & $155,7(134,8-176,6)$ & \\
\hline $80-89$ & $50,0(10,0-180,0)$ & & $147,5(127,9-167,2)$ & \\
\hline 90 o más & $48,0(12,0-108,0)$ & & $188,3(75,2-301,5)$ & \\
\hline Estado civil & & 0,026 & & \\
\hline Casado(a) o conviviente & $35,0(9,0-130,0)$ & & $151,3(136,7-165,9)$ & \\
\hline Soltero(a) & $38,0(8,5-140,0)$ & & $124,9(90,0-159,8)$ & \\
\hline Viudo(a), divorciado(a) o separado(a) & $30,0(10,0-122,5)$ & & $126,7(113,9-139,4)$ & \\
\hline Área de residencia & & $<0,001$ & & $<0,001$ * \\
\hline Urbano & $40,0(10,0-150,0)$ & & $154,8(142,8-166,8)$ & \\
\hline Rural & $13,0(5,0-50,0)$ & & $70,5(60,4-80,7)$ & \\
\hline Nivel educativo & & $<0,001$ & & $<0,001^{\dagger}$ \\
\hline Sin nivel o inicial & $18,0(5,0-70,0)$ & & $68,0(57,7-78,4)$ & \\
\hline Primaria & $25,0(7,0-100,0)$ & & $108,5(97,6-119,3)$ & \\
\hline Secundaria & $42,0(12,0-156,0)$ & & $155,0(134,5-175,5)$ & \\
\hline Superior & $78,0(20,0-242,0)$ & & $247,9(210,5-285,3)$ & \\
\hline Tipo de seguro de salud & & $<0,001$ & & \\
\hline Sin seguro & $30,0(9,0-130,0)$ & & $143,6(118,2-168,9)$ & \\
\hline FF.AA. & $120,0(30,0-225,0)$ & & $213,1(158,3-267,9)$ & \\
\hline SIS & $20,0(6,0-70,0)$ & & $80,8(72,0-89,6)$ & \\
\hline EsSalud & $60,0(15,0-200,0)$ & & $193,2(172,5-213,9)$ & \\
\hline Privado o EPS & $85,0(30,0-300,0)$ & & $249,9(140,4-359,5)$ & \\
\hline Dominio geográfico & & $<0,001$ & & $<0,001^{\dagger}$ \\
\hline Lima Metropolitana & $60,0(15,0-191,0)$ & & $182,7(159,8-205,7)$ & \\
\hline Resto de costa & $32,0(10,0-121,5)$ & & $131,9(117,5-146,2)$ & \\
\hline Sierra & $18,0(6,0-78,0)$ & & $99,8(87,5-112,0)$ & \\
\hline Selva & $24,5(6,0-80,0)$ & & $109,7(87,6-131,8)$ & \\
\hline Enfermedades crónicas & & $<0,001$ & & $0,015^{*}$ \\
\hline Sí & $40,0(10,0-150,0)$ & & $146,7(135,7-157,7)$ & \\
\hline No & $19,3(5,5-75,0)$ & & $113,3(88,3-138,4)$ & \\
\hline Limitaciones físicas o psicológicas & & 0,142 & & $0,367^{*}$ \\
\hline Sí & $33,0(9,0-125,0)$ & & $139,1(127,7-150,5)$ & \\
\hline No & $40,0(10,0-150,0)$ & & $150,7(128,6-172,7)$ & \\
\hline Quintiles de gasto per cápita & & $<0,001$ & & $<0,001+$ \\
\hline Quintil 1 (menor) & $10,0(4,2-30,0)$ & & $35,7(29,8-41,5)$ & \\
\hline Quintil 2 & $17,0(5,5-55,0)$ & & $59,0(52,2-65,7)$ & \\
\hline Quintil 3 & $30,0(10,0-100,0)$ & & $86,9(77,5-96,3)$ & \\
\hline Quintil 4 & $50,0(15,0-180,0)$ & & $166,0(142,9-189,0)$ & \\
\hline Quintil 5 (mayor) & $120,0(35,0-304,0)$ & & $293,3(260,8-325,8)$ & \\
\hline \multicolumn{5}{|c|}{$\begin{array}{l}\text { Las estimaciones incluyeron el factor de expansión y especificaciones muestrales de la ENAHO } 2017 \\
\text { * Significancia estadística obtenida mediante la prueba t de Student } \\
\text { + Significancia estadística obtenida mediante la prueba de ANOVA } \\
\text { ₹ IC } 95 \% \text { calculado mediante bootstrapping con el método basado en la normal con } 1000 \text { replicaciones } \\
\text { Q1-Q3: percentil } 25 \text { - percentil } 75 \\
\text { FF.AA.: Sanidad de las Fuerzas Armadas y Policía Nacional } \\
\text { SIS: Seguro Integral de Salud } \\
\text { EsSalud: Seguro Social } \\
\text { EPS: Entidades Prestadoras de Salud } \\
\text { Tasa de cambio (S/ por USD): } 3,26\end{array}$} \\
\hline
\end{tabular}


bivariado, todos los factores resultaron estadísticamente significativos, a excepción del factor padecer una limitación física o psicológica ( $p=0,641)$. En el modelo multivariado se encontró que quienes tienen educación superior ( $R P a 1,09$; IC 95\%: 1,00-1,18) o padecen una enfermedad crónica (RPa 1,44; IC 95\%: 1,37-1,52) tienen más probabilidades de realizar GBS en comparación a quienes tienen menor educación o no padecen una enfermedad crónica. Asimismo, los adultos mayores en el mayor quintil de gasto per cápita tienen aproximadamente 1,63 veces más probabilidades de realizar GBS en comparación a los del menor quintil gasto per cápita.

Con relación a los niveles medios de GBS, se encontró que los adultos mayores con educación superior tienen un GBS medio de 180,0 soles (USD 55,2) superior a quienes tienen niveles iniciales de educación o no tienen nivel alguno (Tabla 3). Los adultos mayores que padecen enfermedades crónicas destinan aproximadamente 33,0 soles más (USD 10,1) en GBS que quienes no padecen enfermedades crónicas. Por otro lado, estar afiliado al SIS reduce 63,0 soles (USD 19,3) el GBS comparado con aquellos sin seguro de salud. Asimismo, se encontró que los adultos mayores del mayor quintil de gasto per cápita destinan 257,6 soles (USD 79,0) más en GBS frente a los del menor quintil (Tabla 3).

\section{DISCUSIÓN}

Nuestro estudio sobre GBS muestra que la proporción de adultos mayores peruanos que efectuó GBS en 2017 es de $56,5 \%$ y que la media de gasto fue de 140,8 soles (USD 43,2). Esta media representa aproximadamente el $15,1 \%$ de la remuneración mínima vital ${ }^{22}$, una tercera parte de la pensión mínima en Perú ${ }^{(23)}$ y $20,0 \%$ de la línea de pobreza extrema ${ }^{(24)}$. Asimismo, quienes presentan mayores proporciones son los que están afiliados a un seguro privado o EPS y quienes están en el mayor quintil de gasto. Se reportaron diferencias significativas en el nivel de GBS entre los dominios. También se encontró que ser de procedencia urbana, tener mayor nivel de educación, padecer una enfermedad crónica y estar en el máximo quintil de gasto per cápita aumentan el GBS.

En general, las mayores proporciones con GBS se agrupan en adultos mayores con menor desventaja social. Este segmento de adultos mayores tiene mayor riesgo de endeudarse debido a las mayores proporciones de GBS ${ }^{(25)}$. A su vez, quienes presentan menores proporciones con GBS son socialmente vulnerables (adultos mayores sin nivel de educación, de procedencia rural o del quintil menor de gasto per cápita), lo que refleja los obstáculos para acceder al servicio de salud, dada las consecuencias financieras y empobrecedoras que representa el GBS. Los esfuerzos para reducir las desigualdades y garantizar la universalidad de servicios de salud deben priorizar a este último grupo de adultos mayores.

También se encontró que la procedencia urbana, tener mayor nivel de educación, padecer una enfermedad crónica, así como, tener mayores gastos per cápita aumentan las posibilidades y los niveles de GBS. Estos hallazgos son consistentes con los resultados de Bock et al. ${ }^{(6)}$, Gong et al. ${ }^{(7)}$, Turi et al. ${ }^{(10)}$ y Corrieri et al. ${ }^{(13)}$. La fuerte asociación reportada entre el nivel de GBS y los quintiles de gasto per cápita sugiere que la proporción GBS/gasto per cápita es desfavorable para los socialmente desaventajados, como Proaño y Bernabé lo señalan en su estudio ${ }^{(26)}$. Investigaciones de tipo longitudinal se requieren para evaluar a fondo los efectos del GBS en el riesgo financiero de los adultos mayores.

Los adultos mayores que padecen enfermedades crónicas destinan aproximadamente 33 soles más (USD 10,1) al GBS. Debido a que en Perú la prevalencia de las enfermedades crónicas está en crecimiento ${ }^{(27)}$ y que su tratamiento requiere de medicamentos costosos y prolongados en el tiempo, la posibilidad de incurrir en riesgos financieros en pacientes con enfermedades crónicas es alta. El sistema de salud puede diseñar programas enfocados en pacientes crónicos para fortalecer el sistema de vigilancia y la detección temprana de tales enfermedades, con el objetivo de salvaguardar la situación financiera de los adultos mayores.

Los mayores niveles de GBS en adultos mayores que tienen seguros de las FF.AA. o EsSalud se pueden explicar por la insatisfacción del servicio de salud prestado, así como largos tiempos de espera ${ }^{(28)}$. Como consecuencia los pacientes no suelen utilizar este servicio, incentivando a pagar por servicios privados ${ }^{(29)}$. Otros estudios coinciden en que algunos seguros no protegen adecuadamente a la población asegurada ${ }^{(8,30)}$. Otro estudio coincide que en Perú el uso de servicios de salud es diferente según la tenencia y el tipo de seguro ${ }^{(29)}$. Los resultados presentados indican que el SIS reduce el nivel de GBS. Una posible explicación para esto es que los afiliados al SIS, son personas en desventaja social que frecuentemente usan los servicios de salud y reciben lo establecido en el Plan Esencial en Aseguramiento en Salud (PEAS) y prestaciones extras para el cáncer (Plan Esperanza). A su vez, a través del Fondo Intangible Solidario en Salud reciben financiamiento para otras enfermedades de alto costo ${ }^{(31)}$.

Una limitación del presente estudio es que, dada la naturaleza transversal de la encuesta, no se puede establecer causalidad entre las variables de interés y son inherentes los errores de medición propios que podría tener la encuesta. De igual manera, se debe considerar que los pagos desembolsados por los servicios de salud pueden presentar niveles individuales, familiares o una combinación de ambos. Otra limitación es que, las preguntas sobre los pagos desembolsados por los servicios de salud no tuvieron 


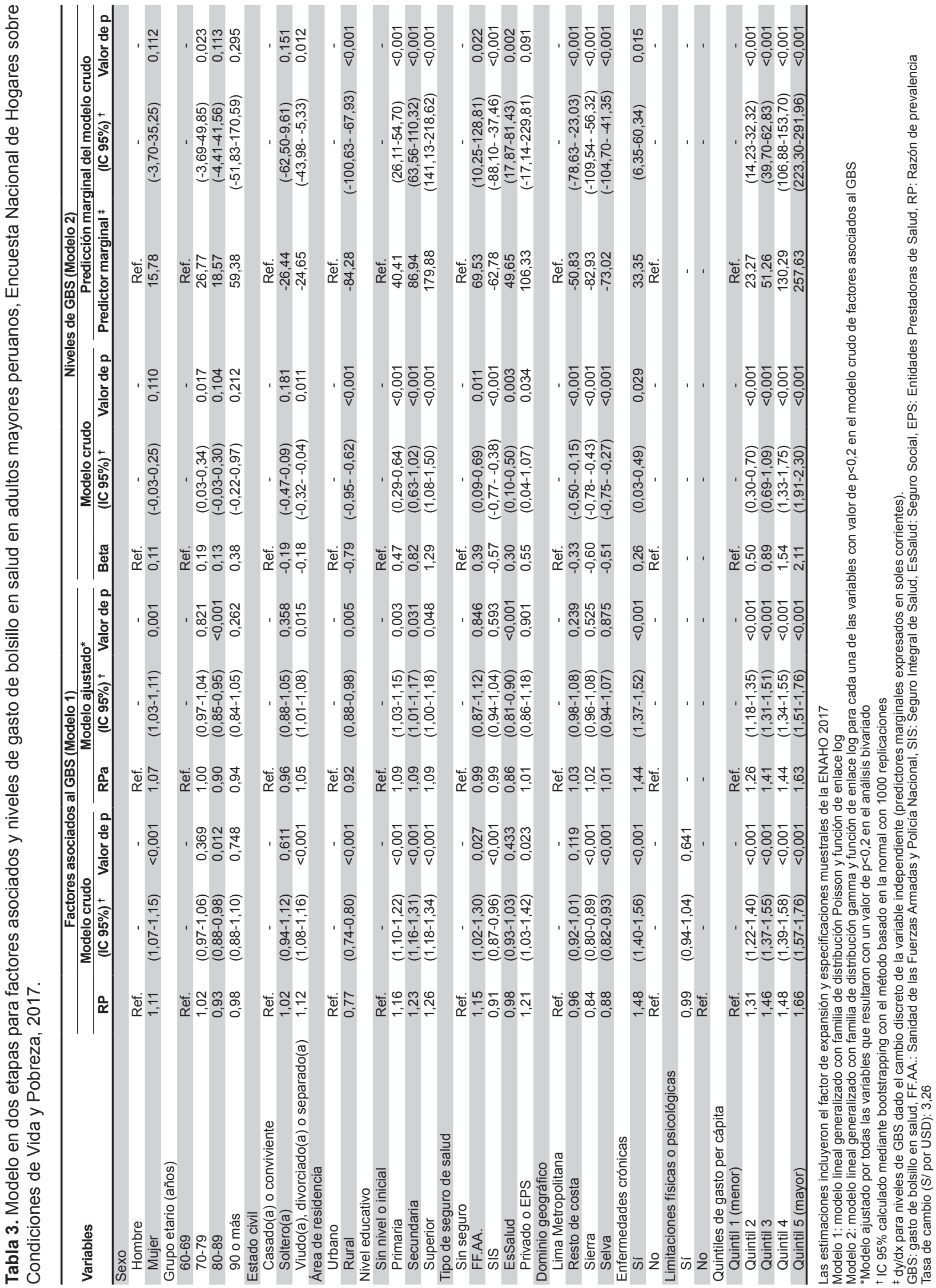


la misma referencia temporal lo que incrementa el riesgo a incurrir en sesgo de recuerdo. Sin embargo, la ENAHO está diseñada para cuantificar el gasto de consumo y caracterizar las condiciones de vida de la población peruana aportando información a nivel poblacional que no se puede conseguir de otras fuentes, por lo tanto, es la única fuente de información que se dispone actualmente en el Perú para realizar análisis de GBS.

En conclusión, los adultos mayores en Perú pagan de su bolsillo para atender su salud, lo que genera inequidad en el acceso a los servicios de salud, principalmente para los más vulnerables, como son la población con menor nivel educativo, menor gasto per cápita, o que padecen enfermedades crónicas. EI SIS ofrece una mayor protección financiera en los adultos mayores. A partir de los resultados obtenidos, se sugiere investigar el impacto económico de los seguros sanitarios y el abordaje preventivo-promocional de las enfermedades crónicas en aras de reducir el GBS y mejorar la eficiencia del sistema de salud peruano.

Contribuciones de autoría: AHV tuvo la idea de investigación, diseñó el estudio, recopiló y procesó los datos. AHV y CRR analizaron los datos. Todos los autores participaron en la interpretación de los datos, redacción del manuscrito y aprobaron la versión final.

Fuentes de financiamiento: autofinanciado.

Conflictos de interés: los autores declaran no tener conflictos de interés.

\section{REFERENCIAS BIBLIOGRÁFICAS}

1. World Health Organization. Out-ofpocket payments, user fees and catastrophic expenditure [Internet]. Geneva: World Health Organization; 2018 [citado el 2 de julio de 2018]. Disponible en: http://www.who.int/health_financing/ topics/financial-protection/out-of-pocket-payments/en/

2. World Health Organization. Global Health Expenditure Database [Internet]. [citado el 2 de julio de 2018]. Disponible en: http://apps.who.int/nha/database/ Select/Indicators/en

3. Sum G, Hone T, Atun R, Millett C, Suhrcke M, Mahal A, et al. Multimorbidity and out-of-pocket expenditure on medicines: a systematic review. BMJ Glob Health. 2018;3(1):e000505. Disponible en: http://dx.doi.org/10.1136/ bmjgh-2017-000505

4. Cohen SB, Uberoi N. Differentials in the Concentration in the Level of Outof-Pocket Health Expenditures across Population Subgroups in the U.S., 2011. In: Statistical Brief (Medical Expenditure Panel Survey (US)) [Internet]. Rockville (MD): Agency for Healthcare Research and Quality (US); 2013. Disponible en: https://www.ncbi.nlm.nih.gov/ pubmed/29708705

5. Mahumud RA, Sarker AR, Sultana M, Islam Z, Khan J, Morton A. Distribution and Determinants of Out-of-pocket Healthcare Expenditures in Bangladesh. J Prev Med Public Health. 2017;50(2):91-9. doi: 10.3961/jpmph.16.089.

6. Bock J-O, Matschinger $\mathrm{H}$, Brenner $\mathrm{H}$, Wild B, Haefeli WE, Quinzler R, et al. Inequalities in out-of-pocket payments for health care services among elderly
Germans--results of a population-based cross-sectional study. Int J Equity Health. 2014;13:3. doi: 10.1186/1475-9276-13-3.

7. Gong $\mathrm{CH}$, Kendig $\mathrm{H}$, He X. Factors predicting health services use among older people in China: An analysis of the China Health and Retirement Longitudinal Study 2013. BMC Health Serv Res [Internet]. 2016;16:63. Disponible en: doi: 10.1186/s12913-016-1307-8.

8. Baird K. High Out-of-Pocket Medical Spending among the Poor and Elderly in Nine Developed Countries. Health Serv Res. 2016;51(4):1467-88. doi: 10.1111/1475-6773.12444.

9. Molla AA, Chi C, Mondaca ALN. Predictors of high out-of-pocket healthcare expenditure: an analysis using Bangladesh household income and expenditure survey, 2010. BMC Health Serv Res [Internet]. 2017;17(1):94. doi: 10.1186/ s12913-017-2047-0.

10. Turi BC, Codogno JS, Sarti FM, Anokye NK, Fernandes RA, Monteiro HL. Determinants of outpatient expenditure within primary care in the Brazilian National Health System. Sao Paulo Med J [Internet]. 2017;135(3):205-12. doi: 10.1590/1516-3180.2016.0224141116.

11. Onwujekwe O, Hanson $\mathrm{K}$, Ichoku $\mathrm{H}$, Uzochukwu B. Financing incidence analysis of household out-of-pocket spending for healthcare: getting more health for money in Nigeria? Int J Health Plann Manage. 2014;29(2):e174-85. doi: 10.1002/hpm.2166.

12. You X, Kobayashi Y. Determinants of out-of-pocket health expenditure in China: analysis using China Health and $\mathrm{Nu}-$ trition Survey data. Appl Health Econ
Health Policy. 2011;9(1):39-49. doi: 10.2165/11530730-000000000-00000.

13. Corrieri S, Heider D, Matschinger $\mathrm{H}$, Lehnert T, Raum E, König H-H. Income-, education- and gender-related inequalities in out-of-pocket health-care payments for $65+$ patients - a systematic review. Int J Equity Health. 2010;9:20. doi: $10.1186 / 1475-9276-9-20$

14. Instituto Nacional de Estadística e Informática. Perú: Crecimiento y distribución de la población, 2017 [Internet]. Lima: INEI; 2018 Jun [citado el 2 de julio de 2018]. Disponible en: https://www. inei.gob.pe/media/MenuRecursivo/publicaciones_digitales/Est/Lib1530/libro. pdf

15. Ley de la Persona Adulta Mayor, Ley No.30490. Diario El Peruano. Jul 20; 2016.

16. Ronald A, Newman JF. Societal and Individual Determinants of Medical Care Utilization in the United States. Milbank Q. 2005;83(4). doi: 10.1111/j.14680009.2005.00428.x.

17. Lazo-González O, Alcalde-Rabanal J, Espinosa-Henao O. El sistema de salud en Perú: situación y desafíos [Internet]. Lima: Colegio Médico del Perú; 2016. Disponible en: http://cmp.org.pe/wp-content/uploads/2018/05/libroSistemaSaludPeru-.pdf

18. Barros AJD, Hirakata VN. Alternatives for logistic regression in cross-sectional studies: an empirical comparison of models that directly estimate the prevalence ratio. BMC Med Res Methodol. 2003;3:21. doi: 10.1186/1471-2288-3-21.

19. Malehi AS, Pourmotahari F, Angali KA. Statistical models for the analysis of skewed 
healthcare cost data: a simulation study. Health Econ Rev. 2015;5:11. doi: 10.1186/ s13561-015-0045-7.

20. Banco Central de Reserva del Perú. Tipo de cambio nominal [Internet]. BCRPData. [citado el 2 de julio de 2018]. Disponible en: https://estadisticas.bcrp.gob.pe/estadisticas/series/mensuales/tipo-de-cambio-nominal

21. Drummond MF, Drummond MF, McGuire A. Economic Evaluation in Health Care: Merging Theory with Practice. New York: Oxford University Press; 2001.

22. Banco Central de Reserva del Perú. Remuneración mínima vital - nominal (S/) [Internet]. BCRPData [citado el 2 de julio de 2018]. Disponible en: https://estadisticas.bcrp.gob.pe/estadisticas/series/mensuales/resultados/PN02124PM/html

23. Oficina de Normalización Previsional [Internet]. Lima: ONP [citado el 2 de julio de 2018]. Sistema previsional y sus actores [aproximadamente 2 ventanas]. Disponible en: https://www.onp.gob.pe/pensiones_ peru_onp/sistema_previsional

24. Instituto Nacional de Estadística Informatica. Evolución de la Pobreza Monetaria en el
Perú, 2016 [Internet]. Lima:INEI; 2017. Disponible en: https://www.inei.gob.pe/ media/cifras_de_pobreza/evolucion-de-lapobreza-2016-10-de-mayo-presentacion. pdf

25. Van Damme W, Van Leemput L, Por I, Hardeman W, Meessen B. Out-of-pocket health expenditure and debt in poor households: evidence from Cambodia. Trop Med Int Health. 2004;9(2):273-80.

26. Proaño FalconiD, BernabéE. Determinants of catastrophic healthcare expenditure in Peru. Int J Health Econ Manag. 2018;9. doi: 10.1007/s10754-018-9245-0.

27. GBD 2016 DALYs and HALE Collaborators. Global, regional, and national disability-adjusted life-years (DALYs) for 333 diseases and injuries and healthy life expectancy (HALE) for 195 countries and territories, 1990-2016: a systematic analysis for the Global Burden of Disease Study 2016. Lancet. 2017;390(10100):1260-344. doi: 10.1016/S0140-6736(17)32130-X.

28. Gallo A, Valdivia G, Soria T. Factores asociados al nivel de satisfacción de los usuarios de consulta externa de diferentes Instituciones Prestadoras de Servicios de Salud (MINSA, EsSalud, FFAA/PNP y clínicas privadas) del Perú en el año 2014 [Tesis]. Lima: Universidad Peruana de Ciencia Aplicadas; 2017.

29. Ypanaqué-Luyo P, Martins M. Uso de los servicios de salud ambulatorios en la población peruana. Rev Peru Med Exp Salud Publica. 2015;32(3):464-70.

30. Pavón-León P, Reyes-Morales H, Martínez AJ, Méndez-Maín SM, Gogeascoechea-Trejo MDC, Blázquez-Morales MSL. Gasto de bolsillo en adultos mayores afiliados a un seguro público de salud en México. Gac Sanit. 2017;31(4):286-91. doi: 10.1016/j.gaceta.2016.12.015.

31. SIS [Internet]. Lima: Seguro Integral de Salud; 2018 [citado el 2 de julio de 2018]. Disponible en: http://www.sis.gob.pe/ index.asp

Correspondencia: Akram Abdul Hernández Vásquez Dirección: Universidad San Ignacio de Loyola, Av. La Fontana 550, La Molina, Lima, Perú

Teléfono: (+511) 3851492

Correo electrónico: ahernandez@usil.edu.pe

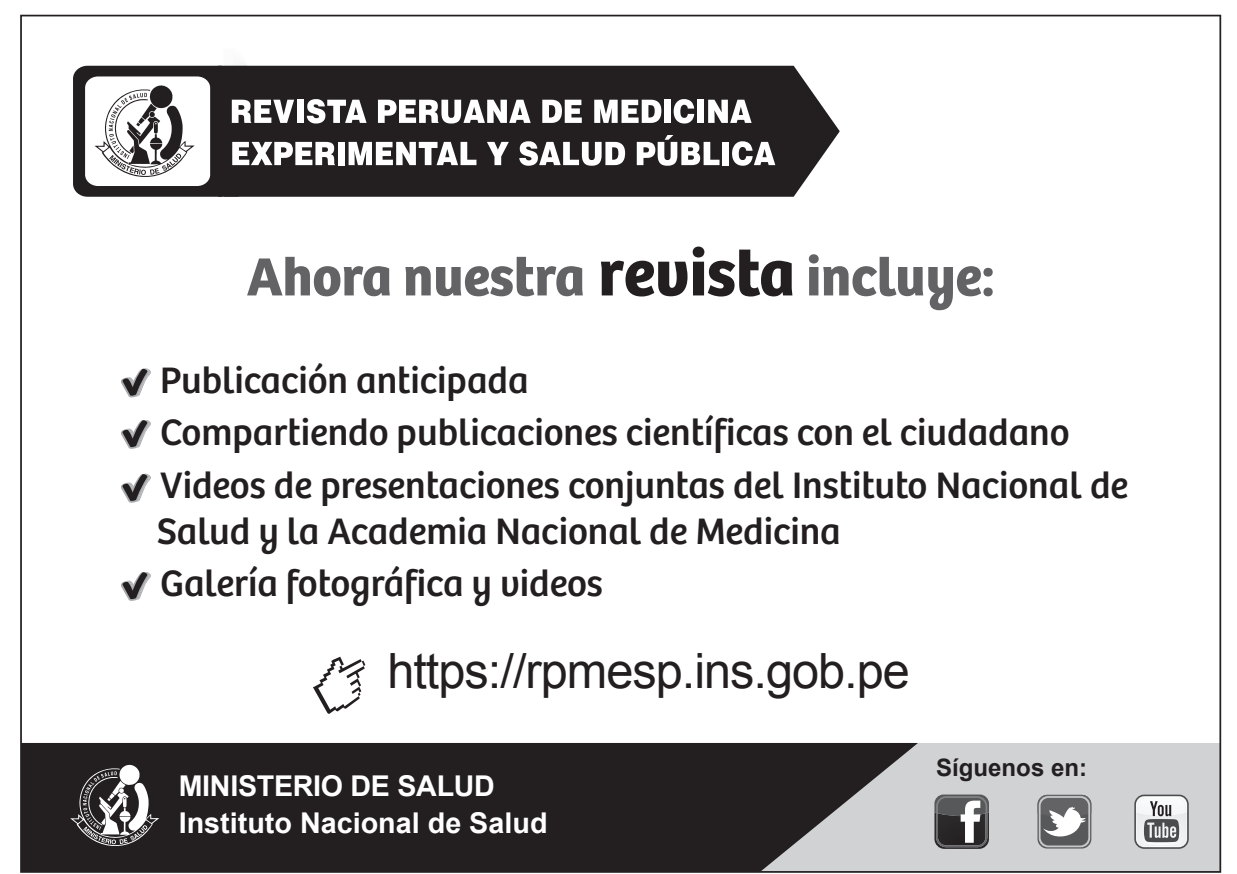

